Textures and Microstructures, Vol. 33, pp. 125-137 Reprints available directly from the publisher Photocopying permitted by license only
(C) 1999 OPA (Overseas Publishers Association) N.V. Published by license under the Gordon and Breach Science Publishers imprint. Printed in Malaysia.

\title{
EXPERIMENTS AND SIMULATIONS BY SELF-CONSISTENT MODELS OF TEXTURE DEVELOPMENT IN Cu-Fe POWDER COMPOSITES
}

\author{
R.E. BOLMARO ${ }^{a}$, A. FOURTY ${ }^{a}$ and H.-G. BROKMEIER ${ }^{b, *}$ \\ ${ }^{a}$ Instituto de Física Rosario-Fac. Ciencias Exactas, Ingeniería y \\ Agrimensura, CONICET-UNR, Bv. 27 de febrero 210 bis. 2000, \\ Rosario, Argentina; ${ }^{b}$ Institut für Metallkunde und Metallphysik der \\ Technische Universität Clausthal, GKSS Forschungszentrum Geesthacht, \\ Max-Planck-Str. 03, D-21502 Geesthacht, Germany
}

Two-Sites Self-Consistent (2-SSC) models have been used for simulation of texture development of two-phase materials (Bolmaro and Lebensohn, 1996; Bolmaro et al., 1997). When both phases are plastically deforming the co-deformation and sharing of spins have to be taken into account via interaction among phases. One-Site Self-Consistent (1-SSC) models are able to consider interaction among each grain and the rest of surrounding grains through a mean field calculated over the "matrix grains". The next level of complexity, i.e., 2-SSC models, is appropriate to capture some interaction features between phases. In the current paper, starting from well-defined microstructures and textures of $\mathrm{Cu}-\mathrm{Fe}$ powder composites, the textures obtained after wire-drawing are simulated taking into account codeformation and sharing of rotations. The results are shown agreeing with experimental ones provided that, depending on volume fraction and relative strength of both phases, different ad-hoc strain rate sensitivities are used.

Keywords: $\mathrm{Cu}-\mathrm{Fe}$ composites; Texture development; Self-consistent simulation; Neutron diffraction

\section{INTRODUCTION}

The purpose of studying texture development in two-phase materials requires reducing the span of materials and regimens under

\footnotetext{
* Corresponding author.
} 
consideration. Being conservative we are talking about:

(a) co-deformable two-phase materials,

(b) yield stress ratios between both phases not larger than 2-3,

(c) no crystallographic correlation between phases,

(d) high volume fractions of both phases.

Even in that scenario there are some experimental results we cannot understand. For instance, the texture of the harder phase is usually stronger than the soft phase texture but sometimes the opposite is true (Brokmeier, 1989; Böcker et al., 1988; Brokmeier et al., 1988; Wassermann et al., 1978; Bolmaro et al., 1994). The first case is more common than the second one and some inconsistencies and questions arise.

(i) If the harder phase always deforms less how does it manage to reach intensities higher than the softer phase?

(ii) Where does the opposite behavior stem from? From crystallinity? From more continuum mechanics considerations?

Another phenomenon that should be explained is that sometimes the texture intensities of harder phases are higher than even the hard phase as a single-phase material. If the soft phase takes part of the deformation of the two-phase composite, it should be taken a large portion of the macroscopic strain, leaving the harder phase with less deformation than if it was single-phase.

In the current paper we do not intend by any means to give a definite explanation to those phenomena. We will merely show some experimental texture results taken by neutron diffraction for wire drawn $\mathrm{Cu}-\mathrm{Fe}$ powder composites. They have been carefully analyzed and freed of inconsistencies in such way that they allow to draw some general conclusions. The wire drawing strain is stopped every 0.25 Von Mises deformation and the texture is measured for the starting material and at every deformation step.

We have also performed simulations by 1-site and 2-sites viscoplastic self-consistent models (2-SVPSC) (Bolmaro et al., 1997). The ability of the last one to consider different stress exponents for each phase has been improved allowing the change of the stress exponents at arbitrarily chosen steps. Preliminary results show the possibilities of the model to explain some of the main experimental results. 


\section{EXPERIMENTAL RESULTS}

\section{Sample Preparation}

The samples were prepared by powder metallurgy. The starting materials were pure powders. On one hand spherical iron particles of $13 \mu \mathrm{m}$ average size and on the other hand dendritic copper powder of which the fraction $<32 \mu \mathrm{m}$ was used (Beusse et al., 1992). Sample preparation starts with homogeneous mixing of $25 \% \mathrm{Cu}$ and $75 \% \mathrm{Fe}$ in a turbula mixer. Thereafter cylinders of diameter $75 \mathrm{~mm}$ are compacted, heated up to $850^{\circ} \mathrm{C}$ and extruded to rods of diameter $19.8 \mathrm{~mm}$. The final state was cold drawing to equivalent Von Mises strains of $0.25,0.50,0.75,1.00$, $1.25,1.50,1.75,2.00,2.25,2.50,2.75$ and 3.00 .

Microstructure, characterized after hot extrusion, shows $\mathrm{Cu}$ dimensions of about $7 \mu \mathrm{m}$ by $40 \mu \mathrm{m}$ in average. Whereas the Fe particles were much smaller and less deformed compared to $\mathrm{Cu}$ showing particles of about $3 \mu \mathrm{m}$ in the short axis and $6 \mu \mathrm{m}$ the long axis in average.

\section{Pole Figure Measurement}

Texture investigations of $\mathrm{Cu}-\mathrm{Fe}$ composites were performed at TEX-2, the texture diffractometer at FRG-1, Geesthacht, Germany (Brokmeier et al., 1998). There are three main reasons to use neutrons instead of $\mathrm{X}$-rays. First is that the neutron technique allows to measure complete pole figures of cylindrical samples. Consequently, corrections are reduced to the correction for background and the results show high accuracy. This high accuracy is necessary to see small differences in the quantitative texture by comparing samples with small differences in the degree of deformation. Second is the sample preparation. All samples have to be cut only to the right length that depends on the diameter of the wire. In the case of low deformation the whole cylindrical sample of about $1000 \mathrm{~mm}^{3}$ in volume was measured. In the case of highly deformed samples the cross section of the wire is rather small. Thus, one is able to produce a composite of three or four sections to increase the effective sample volume and to reduce the counting time. Additional sample preparation, particularly of the sample surface, is not necessary. Third is that iron and copper show similar transmission behavior and absorption correction can be neglected. 
Depending on the wire drawing it would be expected that the texture type is axially symmetric. This was confirmed by the measurement of some complete pole figures. Figure 1(a) shows the counting grid used to cover a complete pole figure and in Fig. 1(b) a complete Fe (110) pole figure of the sample VM 1.75 is given.

To optimize the total counting time for the thirteen samples a rotating sample holder was used and a one-dimensional pole figure was measured. Sample rotation speed was about 80 rotations per second. The step width of the $\alpha$-scan measurement was $5^{\circ}$. From each sample the pole
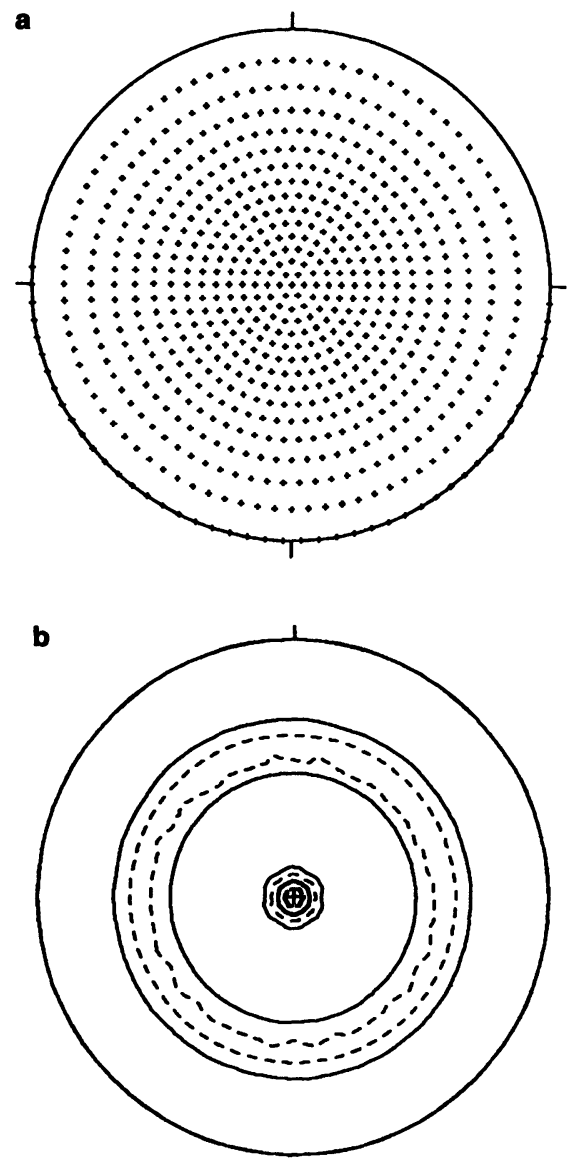

FIGURE 1(a) and (b) 


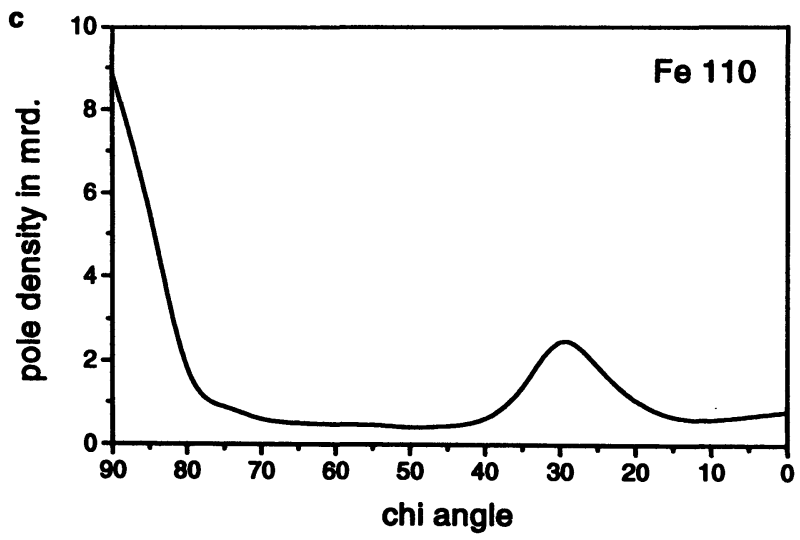

FIGURE 1(c)

FIGURE 1 (a) Equal area grid used for measurements (679 points). (b) $\mathrm{Fe}$ (110) complete pole figure at Von Mises deformation of 1.75. (c) Corresponding $\mathrm{Fe}(110) \alpha$-scan.

TABLE I Experimental conditions of the pole figure measurements on the $\mathrm{Fe}-\mathrm{Cu}$ composites

\begin{tabular}{ll}
\hline Wavelength & $0.133 \mathrm{~nm}$ \\
Monochromator & Cu 111 \\
Inpile collimator & $6 \times 6 \mathrm{~cm}^{2}$ \\
Distance sample-detector & $161 \mathrm{~cm}$ \\
Sample diaphragm PB & $22 \mathrm{~mm}$ \\
Detector diaphragm DB1 & $22 \mathrm{~mm}$ \\
Detector diaphragm DB2 & $40 \mathrm{~mm}$ high, 22 mm wide \\
Counting grid & $\alpha$-scan 19 points rotating sample \\
\hline
\end{tabular}

figures $\mathrm{Cu}$ (111), $\mathrm{Cu}(200), \mathrm{Cu}(220), \mathrm{Fe}(110), \mathrm{Fe}(200)$ and $\mathrm{Fe}(211)$ were measured. The counting time for each pole figure point varied between 40 and $250 \mathrm{~s}$, depending on the expected texture, on the scattering behavior of $\mathrm{Fe}$ and $\mathrm{Cu}$ respectively and on the total sample volume in the beam. In Fig. 1(c) a typical $\alpha$-scan for Fe (110) is shown. The experimental conditions, which are summarized in Table $I$, are constant during the whole experiment.

The data from each $\alpha$-scan were symmetrized to a complete pole figure with an equal angular grid of a $5 \times 5$ matrix and corrected for background. Furthermore, pole figure inversion was carried out by Iterative Series Expansion Method (ISEM) (Dahms and Bunge, 1989) and the WIMV method (Kallend et al., 1991). 


\section{Texture Description}

The starting material for this investigation, the hot extruded rod, shows in the case of $\mathrm{Fe} \mathrm{a}\langle 110\rangle$ fiber texture with an orientation density of about $5.6 \mathrm{mrd}$. In the case of $\mathrm{Cu}$ a double fiber has been developed $(\langle 100\rangle$ with $5.4 \mathrm{mrd}$ and $\langle 111\rangle$ with $2.2 \mathrm{mrd})$. Figure 2 shows the evolution of the main components of $\mathrm{Fe}$ and $\mathrm{Cu}$ in wire direction starting from the asreceived hot-extruded sample until the one for equivalent Von Mises deformation of 3.00 .

In the case of low strains the Fe texture goes down from $5.6 \mathrm{mrd}$ (asreceived hot extruded) to $5.1 \mathrm{mrd}$ at a Von Mises strain of 0.50 . With increasing deformation the Fe texture develops continuously up to a Von Mises strain of 2.5 to a texture sharpness of $11.6 \mathrm{mrd}$. A further increase of the deformation starts a process that leads to a decrease of the texture, see Fig. 2. This process could be curling of $\mathrm{Cu}$ and $\mathrm{Fe}$ particles. At such a high deformation cracking of elongated fiber like particles could also occur. In the case of the $\mathrm{Cu}$ a more complex development can be observed in Fig. 2. The strong $\langle 100\rangle$ fiber of the hot extruded material goes down with increasing deformation. That means the 100 texture component was destroyed by increasing deformation. As shown in Fig. 2 the $\mathrm{Cu} 111$ texture component goes parallel to the $\mathrm{Fe} 110$ texture, but

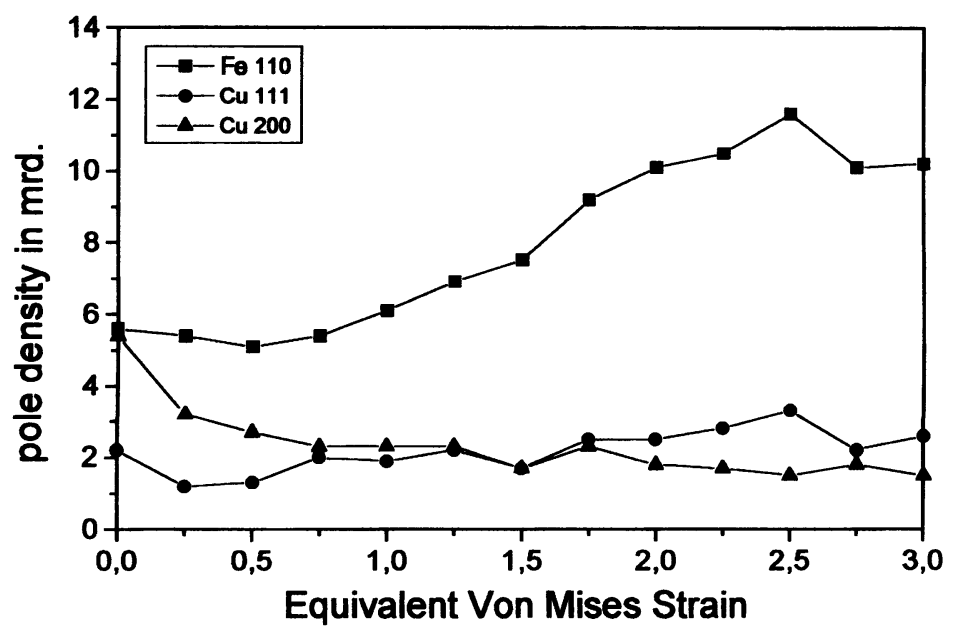

FIGURE 2 Development of texture components in function of the Von Mises equivalent strain. 
on a lower level. First the starting texture $(2.2 \mathrm{mrd})$ was destroyed a little, thereafter a continuous increase of the texture was observed up to Von Mises 2.5 (3.3 mrd) and furthermore the texture goes down slowly. It can be pointed out that the $\mathrm{Cu}$-texture is much weaker than the texture of the harder $\mathrm{Fe}$. One very important characteristic of the $\mathrm{Cu}$ inverse pole figures that cannot be shown by that plot is the continuous switching between the (100) direction and the (111) direction that creates some unstable components between them. Figure 3 shows the inverse pole figures for both phases at Von Mises equivalent strain of 2.00.

The unstable component is clear at around orientation (112). Figure 4 shows the intensities, every $5^{\circ}$, along a section between (100) and (111)

a

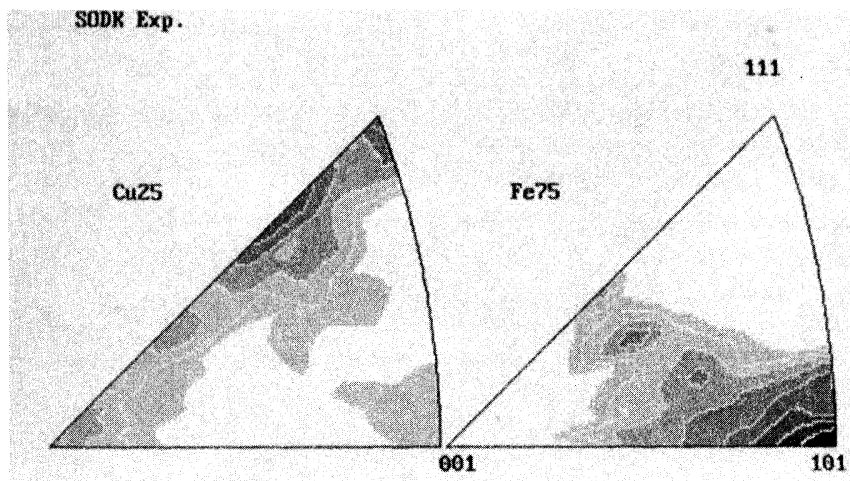

$\operatorname{nax} .=3.63$

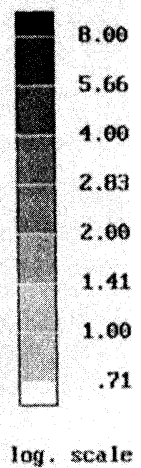

b soDK SIn.

$\max ,=4.61$

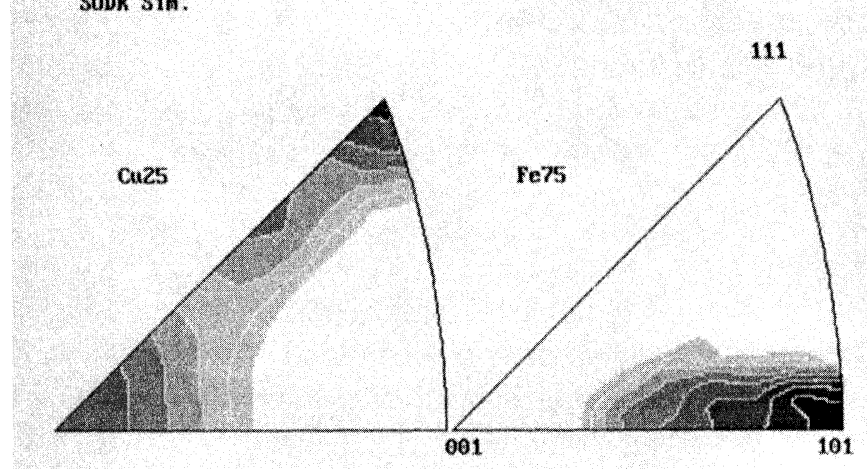

8. 00 5.66 4.00 2.83 2.00 1.41 1.00 .71

FIGURE $3 \mathrm{Cu}$ and $\mathrm{Fe}$ inverse pole figures at Von Mises 2.00. (a) ODF-calculation. (b) Simulated. 


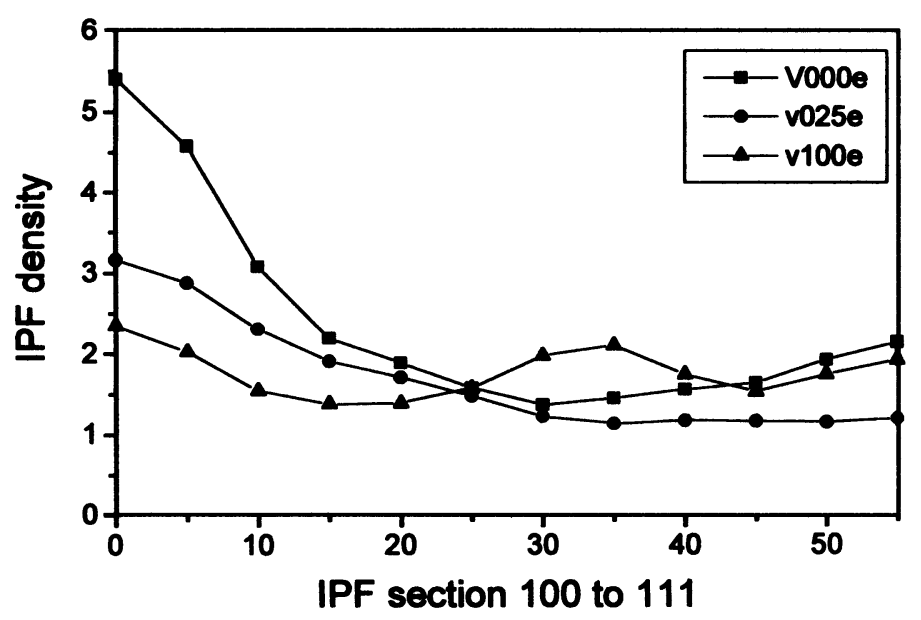

FIGURE 4 Inverse pole figure section from (100) to (111) for $\mathrm{Cu}$ in wire direction: ODF-calculation.

orientations for different strain steps. The continuous pace of transference between both components is clear for increasing strains.

\section{SIMULATIONS}

The main assumptions of the model have been given elsewhere (Bolmaro et al., 1997). Equal sharing of rotations is assumed to hold starting right from the beginning of the deformation process due to the very well elongated and aligned microstructure presented by the starting samples.

The spin sharing equation stems from considering that the spin of both grains included in the original hole can be written as

$$
\Omega_{i j}=\left\langle\Omega_{i j}\right\rangle=\left(\Omega_{i j}^{1 *}+\Omega_{i j}^{2 *}+\Omega_{i j}^{1 \mathrm{P}}+\Omega_{i j}^{2 \mathrm{P}}\right) / 2
$$

where $\Omega_{i j}$ is the external macroscopic spin, assumed constant and equal for every hole apportioned to each pair of grains, $\Omega_{i j}^{k *}$ is the crystal system spin, $\Omega_{i j}^{k \mathrm{P}}$ is the spin of the grain shape due to plastic crystal slip and \langle\rangle means the average magnitude. If the average spin $\left\langle\Omega_{i j}^{*}\right\rangle$ is associated with the back spin of each pair of grains, then

$$
\left\langle\Omega_{i j}^{*}\right\rangle=\left(\Omega_{i j}^{1 *}+\Omega_{i j}^{2 *}\right) / 2=\Omega_{i j}-\left(\Omega_{i j}^{1 \mathrm{P}}+\Omega_{i j}^{2 \mathrm{P}}\right) / 2
$$


and we should use an average grain shape spin (produced by the plastic deformation), for both grains, subtracted from the macroscopic spin.

Co-deforming two-phase materials mean inhomogeneous and nonstationary stress and strain fields. When inhomogeneity is low we can assume it is represented by slight modifications in the constitutive equations. With that assumption the many slip systems activated in different regions of one grain can be considered averaged over the grain. In the current paper we have implemented a systematic search for the best agreement between experimental and simulated textures by varying the $n$ stress exponents for each phase. The agreement has been judged by numerically comparing the distance between both textures in the ODF space every $5^{\circ}$. The distance was calculated by

$$
\sum_{\varphi_{1} \phi \varphi_{2}}\left[I_{\varphi_{1} \phi \varphi_{2}}^{\exp }-I_{\varphi_{1} \phi \varphi_{2}}^{\text {theor }}\right]^{2} /\left(I_{\varphi_{1} \phi \varphi_{2}}^{\exp }\right)^{2}
$$

where $I_{\varphi_{1} \phi \varphi_{2}}^{\exp }$ and $I_{\varphi_{1} \phi \varphi_{2}}^{\text {theor }}$ are the experimental and theoretical intensities for each $\varphi_{1}, \phi, \varphi_{2}$ orientation. Different contour level maps were plotted for many possible combinations of stress exponents. Also some coarse "naked eye" comparison between simulated and experimental textures was done. The results come out to be compatible. The usual disadvantage of numerical comparison between experimental and simulated textures is that any distance definition you can choose tends to overemphasize some a priori chosen characteristic of the pole figures. This is particularly true when simulated textures are far in intensities from the experimental ones and the search is more for matching in components than for the intensities themselves (Signorelli, 1998; Tarasiuk and Wierzbanowski, 1996). Our simulations proved to be close enough in intensities to show no differences whichever mathematical definition of distance is used. Just some small details are lost in some cases and the mathematical approach was better to capture some of them than the pure empirical "naked eye" comparison.

Iron was considered to be harder than copper. The calculations were performed under the assumption of equal spin sharing. We considered 500 grains randomly oriented and randomly related in pairs with other 500 grains of the other phase. The relative size of the grains was weighted to match a distribution compatible with the measured starting texture. The starting shape was introduced resembling the measured aspect ratio 
for both phases. The relative strengths of both phases were parametrized by a starting yield stress for the harder phase (i.e., the iron) of twice the yield stress of the softer one, taken arbitrarily equal to 1 . Common FCC $\{111\}\langle 110\rangle$ and $\mathrm{BCC}\{110\}\langle 111\rangle$ yield behaviors were assumed for $\mathrm{Cu}$ and $\mathrm{Fe}$ respectively. Strain hardening was assumed linear with slopes of 1.5 for $\mathrm{Cu}$ and 2 for $\mathrm{Fe}$ in the same arbitrary units used for critical stresses.

The calculated intensities for the different components of both phase textures are shown in Fig. 3(a) and (b). The inverse pole figures for $\mathrm{Cu}$ and $\mathrm{Fe}$ are calculated, ODF-analysis and simulation, at a Von Mises equivalent deformation of 2.00. In Fig. 5 we show the simulated evolution of the (100) to (111) transference. We see that they come out quite in agreement with the experimental ones. The (112) component has developed quite clearly. It might have the characteristic of an unstable component with many grains reaching and leaving that orientation at each step.

The number of active slip systems are plotted in Fig. 6. At low deformations (between 0 and 0.5 Von Mises) the behavior of the number of slip systems active in $\mathrm{Cu}$ is rather unpredictable. That the number of slip systems is actually quite high may be due to a change from a high temperature regime (high temperature extrusion) to a low temperature

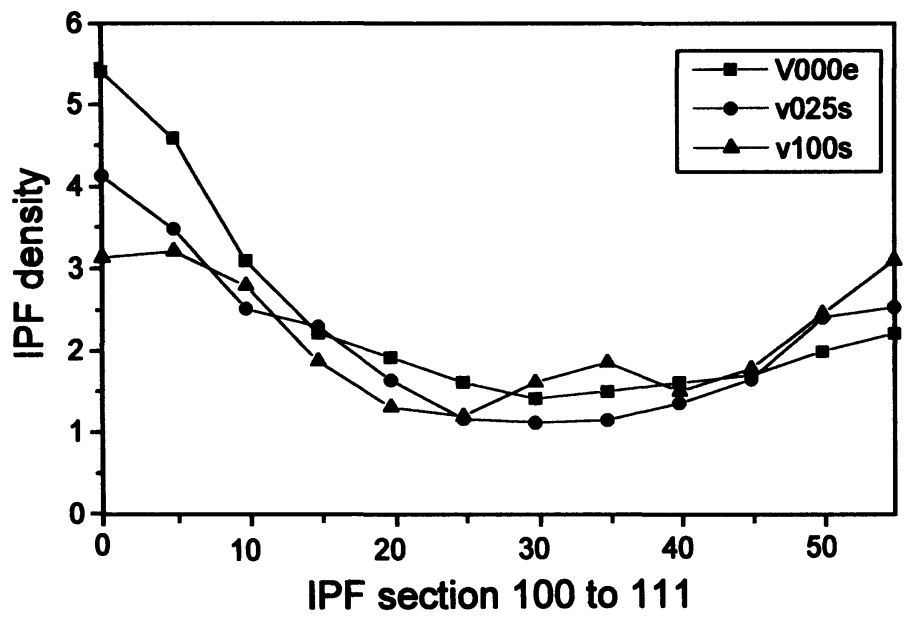

FIGURE 5 Inverse pole figure section from (100) to (111) for $\mathrm{Cu}$ in wire direction: Simulation. 


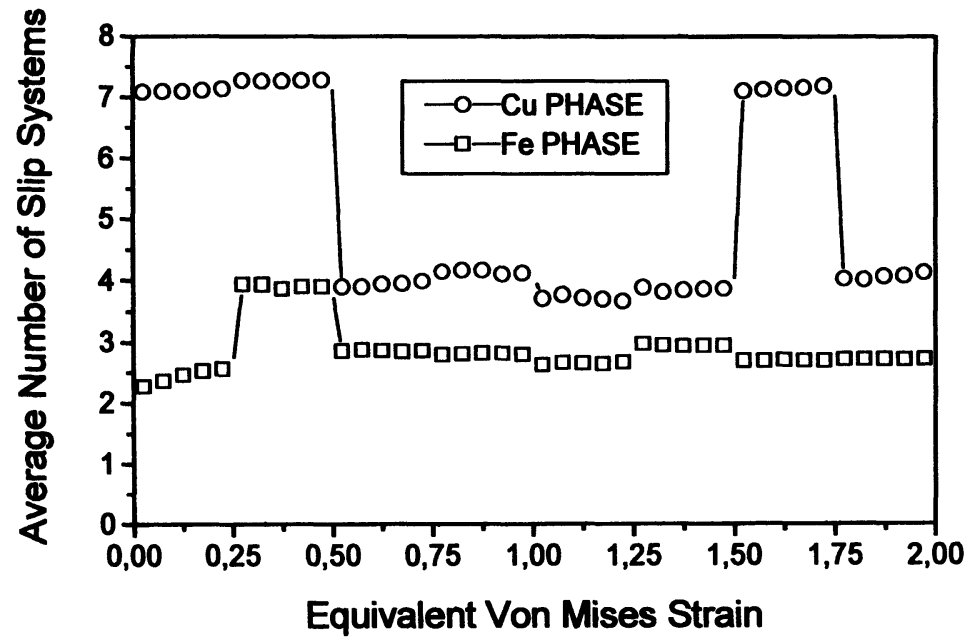

FIGURE 6 Stress exponents used for each phase at each deformation step and the corresponding number of slip systems.

one (wire drawing). Also geometrical re-arrangement of phases could be happening. At a deformation of 1.5 Von Mises there is a sudden increase in the number of slip systems active in $\mathrm{Cu}$. We have already noticed some singular points on the pace of development of the $\mathrm{Cu}(100)$ and (111) and $\mathrm{Fe}$ (110) texture components (Fig. 2). At that stage curling or fiber like grains breaking could be occurring. We found that at low deformations not too much improvement can be achieved by using different stress exponents. The main disadvantage of a 2 -sites simulation at low deformations is that no clear identification can be done of what is called the closest neighbor when the grains are round. When they become elongated clearly the ones with the largest side in contact will influence more the behavior of the neighbor. In fact some preliminary results obtained by 1 -SSC models show slightly better agreement with the experiments at low deformations.

\section{CONCLUSIONS}

Co-deformation induces such interaction between closest neighbors that some amount of sharing of spins has to be enforced. That is one 
of the main ad hoc assumptions allowing to achieve close matching between simulated and experimental textures.

Interaction among grains and phases has a profound effect on the strain rate sensitivities shown by both phases. The number of slip systems steadily grows for higher strains which seems to be compatible with the diminishing aspect ratio of both phases. Modifications of the strain rate sensitivities can always be rationalized in terms of volume fraction (percolation or not) degree of deformation, shape of grains, etc. The variation of stress exponents is not meant to be a description of the real facts but only a physically based numerical trick to enforce different velocity gradient distributions for each phase. In a more detailed model ( $N$-sites approach, FEM based models) that variation should come out naturally from the interaction equations between different grains and phases. In fact, previous calculations performed by finite element models show the effect of strain path changes and fast changing relative spins that conform regions of altered flow patterns of different magnitudes (Bolmaro et al., 1994). When those variations are kept within narrow limits, like in co-deformable materials, the effect can be introduced by modifications of the strain rate sensitivities of both phases.

The evolving textures, measured with high accuracy, can be used to tune the model in order to get information about interaction among phases and degrees of inhomogeneity of the deformation process.

Two-sites self-consistent models are appropriate for texture simulations on at least the very narrow fringe of low inhomogeneity two-phase materials. At low and very high deformations the agreement seems to be lower than at intermediate deformations. The disagreement at low deformations can be due to the poor ability of a 2-site model to identify the main interactions between grains and a failure of the co-rotation scheme. At high strains the whole deformation and interaction scheme can be changed by fracture of $\mathrm{Fe}$ and $\mathrm{Cu}$ particles and by curling effects. That is to say, by a sudden increment of inhomogeneity of the deformation. A new starting, with new microstructure description parameters, should be allowed but the unknowns of the process increase dramatically at this point. The co-rotation scheme can be completely invalidated when the grains acquire a complete new geometry by fracturing or curling.

The problem of interaction between phases is by no means a solved problem: The models have to be continuously checked against 
experiments. Notwithstanding the interaction through the co-rotation assumption seems to be strong enough to be seriously considered in future.

\section{References}

Beusse, R., Böcker, W. and Bunge, H.J. (1992). Scripta Met. 27, 767-770.

Böcker, W., Brokmeier, H.-G. and Bunge, H.J. (1988). Proceedings ICOTOM 9, TMSAIME, Warrendale, USA, pp. 959-964.

Bolmaro, R.E., Browning, R.V., Guerra, F.M. and Rollett, A.D. (1994). Mat. Sci. Eng. A $175,113-139$.

Bolmaro, R.E. and Lebensohn, R.A. (1996). Proceedings ICOTOM 11, Xi'an, China, pp. 1209-1214.

Bolmaro, R.E., Lebensohn, R.A. and Brokmeier, H.-G. (1997). Comp. Mat. Sc. 9, 237-250.

Brokmeier, H.-G. (1989). Textures Microstruc. 10, 325-346.

Brokmeier, H.-G., Böcker, W., Dahms, M. and Bunge, H.J. (1988). 9 th RISO Int. Symp. on Metallurgy and Materials Science, Roskilde Denmark, pp. 309-314.

Brokmeier, H.-G., Zink, U., Schnieber, R. and Witassek, B. (1998). Mat. Sci. Forum 273-275, 277-282.

Dahms, M. and Bunge, H.J. (1989). J. Appl. Cryst. 22, 439-447.

Kallend, J.S., Kocks, U.F., Rollett, A.D. and Wenk, H.-R. (1991). Mat. Sci. Eng. A 132, 1-11.

Signorelli, J. (1998). Ecole nt. Supérieure des Mines de Paris, Rapport DEA, Centre de Mise en Forme des Matériaux, Sophia Antipolis.

Tarasiuk, J. and Wierzbanowski, K. (1996). Phil. Mag. A 73, 1083-1091.

Wassermann, G., Bergmann, H.W. and Frommeyer, G. (1978). Proceedings ICOTOM 5, Aachen, Vol. II, Springer, Berlin, pp. 37-46. 